\title{
Electrophysiological Study of Visual Pathways in Nevoid Basal Cell Carcinoma Syndrome Patients
}

This article was published in the following Dove Press journal: Eye and Brain

\author{
Antonietta Moramarco ${ }^{1, *}$ \\ Ludovico Alisi $\mathbb{D}^{1, *}$ \\ Alessandro Lambiase $\mathbb{D}^{\prime}$ \\ Sandra Giustini $\mathbb{D D}^{2}$ \\ Luca Lucchino' \\ Emanuele Miraglia ${ }^{2}$ \\ Vincenzo Roberti (D) ${ }^{2}$ \\ Marcella Nebbioso (iD) \\ 'Department of Sense Organs, Faculty of \\ Medicine and Odontology, Sapienza \\ University of Rome, Rome, 0016I, Italy; \\ ${ }^{2}$ Department of Dermatology, Sapienza \\ University of Rome, Rome, 00185, Italy \\ *These authors contributed equally to \\ this work
}

Introduction: Gorlin-Goltz syndrome (GGS) also known as nevoid basal cell carcinoma syndrome (NBCCS) is a complex rare genetic disorder characterized by a wide range of clinical and radiological manifestations. Ophthalmological alterations have always been reported, but no study on the eventual pattern visual evoked potentials (pVEPs) abnormalities has yet been published.

Purpose: The purpose of the study was to evaluate the functionality of the optic pathways in a group of NBCCS patients through pattern reversal VEPs, after a thorough exclusion of subjects with preexisting ocular and optic pathways pathologies.

Methods: Nineteen NBCCS patients (31 eyes) and 20 healthy controls (40 eyes) have been recruited for this study. All subjects underwent an evaluation of the functionality of the optic pathways through pVEPs with small $\left(120^{\prime}\right)$, medium $\left(60^{\prime}\right)$, and large $\left(15^{\prime}\right)$ check size stimulation.

Results: NBCCS patients showed a statistically significant alteration in the transmission of the macular pathway function when compared to controls. PVEPs analysis confirmed a reduced amplitude and an increased latency of the P100 component, suggesting an involvement of the visual pathway even in the absence of ocular clinical manifestations.

Conclusion: Visual pathways may have been affected both by a subclinical myelination deficit, determined directly by the genetic alteration, as well as by neurological abnormalities typical of this syndrome. Further studies are warranted.

Keywords: Gorlin-Goltz syndrome, nevoid basal cell carcinoma syndrome, pattern visual evoked potentials, rare diseases, electrophysiology, visual pathway, genodermatosis, PATCH1

\section{Introduction}

In 1960 Robert Gorlin and Robert Goltz first described a syndrome characterized by nevoid basal cell epithelioma, jaw cysts, and bifid ribs, nowadays known as GorlinGoltz syndrome (GGS) or nevoid basal cell carcinoma syndrome (NBCCS). ${ }^{1}$ NBCCS (OMIM 109400) is an autosomal dominant disease with near complete penetrance and variable expressivity. NBCCS is considered a rare disease and its prevalence, similar in men and women, is estimated between $1 / 57.000$ to $1 / 256.000$ with significant differences between races and ethnicity. ${ }^{2,3}$ This genodermatosis is determined by a mutation of the PATCH1 gene located on chromosome 9 q22.3-q31. PATCH1 acts as a tumor suppressor gene and codes for the transmembrane glycoprotein Patched 1. Patched 1 plays a cardinal role in numerous pathways as it binds transcription factors such as Sonic Hedgehog (SHH). The most frequent mutations are insertion or deletion, but nonsense, missense, and splicing mutations
Correspondence: Alessandro Lambiase University of Rome, Viale del Policlinico I55, Rome, 0016I, Italy

Tel +3906 49975357

Fax +390649975425

Email alessandro.lambiase@uniromal.it 
have been reported as well. ${ }^{4}$ Mutations of the PATCH1 gene are responsible for about $50-85 \%$ of the cases of NBCCS with higher estimates closer to $90 \%$ in more recent studies. ${ }^{5}$ The loss of function of PATCH1 determines the unchecked activation of cellular proliferative pathways. ${ }^{6}$ Recently two other genes have been recognized responsible for the development of NBCCS. PTCH2, highly homologous to PTCH1, located on chromosome 1p32.1-p32.3 and suppressor of the fused gene (SUFU) located on chromosome 10q. ${ }^{7,8}$ Interestingly, patients with SUFU mutation show an increased incidence of medulloblastoma when compared to patients carrying PATCH1 mutation. ${ }^{9}$

NBCCS is a complex syndrome with more than 100 recognized clinical manifestation. Cardinal features are multiple basal cells carcinoma (BCC) that usually develop around the second or third decade but have reported in children as well; ${ }^{10}$ keratocystic odontogenic tumor, usually multiple lesions can be found in $90 \%$ of the affected patients above the age of $40 ;{ }^{11}$ ribs or spine abnormalities such as bifid ribs and early calcification of the falx cerebri. $^{12,13}$

Major diagnostic criteria include multiple $(>2)$ BCCs or $1 \mathrm{BCC}$ by $\leq 30$ years of age, or more than 10 basal cell naevi, any odontogenic keratocysts of the jaw proven by histology, palmar or plantar pitting, lamellar calcification of the falx cerebri and first-degree relative with NBCCS. Minor diagnostic criteria include congenital skeletal anomalies such as bifid, fused or missing ribs, medulloblastoma, ovarian and cardiac fibromas, lymphomesenteric cysts, increased circumference of the head, and other congenital malformations (frontal bossing, coarse facies, cleft lip or palate, moderate or severe hypertelorism, etc.). ${ }^{10}$ Diagnosis requires the concomitant presence of 2 major criteria and 1 minor criterion or 1 major criterion and 3 minor criteria. Oftentimes though, in developed countries diagnosis is achieved by genetic analysis on the PTHC1 gene or the other involved genes. ${ }^{13}$

Numerous ocular manifestations have been reported in patients with NBCCS with a higher prevalence compared to the general populations. Most of these reports are sporadic and confined to case reports as only a few works have considered ocular manifestations of NBCCS.

Black et al demonstrated in 30 NBCCS patients the tendency to develop fibroglial epiretinal membrane (ERM), abnormal ganglion-cell axon myelination due to the inactivating mutation of the PATCH1 gene. ${ }^{14}$ Other common findings in NBCCS patients are hypertelorism, congenital cataract, strabismus, myelinated fibers, retinal hamartoma, optic nerve colobomas, nystagmus and palpebral ptosis. $^{15-18}$

The objective of the present study is to evaluate the integrity of the macular pathways and functionality in NBCCS patients by means of pattern reversal visual evoked potentials (pVEPs). The results obtained were compared with a control group (CG) of healthy subjects. In literature, there is only a single case report that studied pattern VEP changes in a patient with GGS, which found reduced amplitude in both eyes and delayed latency in one eye. ${ }^{19}$

\section{Materials and Methods}

We recruited 19 patients affected by NBCCS for a total of 37 eyes ( 1 patient suffered from congenital unilateral anophthalmia) and 20 age-matched healthy controls for a total of 40 eyes. The two groups also matched for ethnicity (Caucasian) and sex. The NBCCS group consisted of 19 subjects 13 females and 6 males with a mean age of 45.5 years \pm 20.2 standard deviation (SD). NBCCS diagnosis was performed according to the Kimonis's diagnostic criteria (two major criteria or one major and two minor criteria) during the dermatological exam that preceded the recruitment in the present study. ${ }^{20}$ Moreover, the molecular testing showed the variant of the PTCH1 gene in every patient included in the NBCCS group.

CG consisted of 20 subjects, 15 females, and 5 males (mean age $46.6 \pm 13.6 \mathrm{SD}$ ). Controls showed no manifest ocular pathology, nor systemic pathologies that could have altered the outcome of the study.

The enrollment took place between May 2019 and February 2020 at the Umberto I Policlinico. The study protocol was approved by the Ethics Committee of the Sapienza University of Rome. The research followed the tenets of the Declaration of Helsinki and informed consent was obtained by all the participants before the enrollment.

Both groups underwent complete ophthalmological examination that included: monocular best-corrected visual acuity (BCVA) measured to 5 meters with Snellen chart; intraocular pressure measurement with Goldman applanation tonometer; anterior segment biomicroscopy and mydriatic indirect fundus biomicroscopy. Furthermore, all patients and controls were examined with spectral-domain optical coherence tomography 
(SD-OCT; Spectralis Family Acquisition Module, V5.1.3.0; Heidelberg Engineering with Heidelberg Eye Explorer V1.6.2.0).

In the NBCCS group, we excluded from the study eyes with visual acuity less than $10 / 10(\log M A R>0.00)$, refractive defects greater than \pm 4 diopters (spherical equivalent), and simultaneous ocular pathologies such as glaucoma, epiretinal membrane, macular pucker, optic nerve coloboma, or cataract. This led to the exclusion of 6 eyes, therefore only 31 eyes in the NBCCS group were considered for both the statistical analysis and pVEPs recording, while all 40 eyes in the control group underwent pVEPs examination.

To record the pVEPs we placed 1 midline active skinelectrode on the scalp over the visual cortex at $\mathrm{O}_{z}$ with the reference electrode at $F_{z}$ on the forehead, and 1 neutral electrode on the earlobe of the subjects. Each eye was examined separately at $100 \mathrm{~cm}$ with the optoelectronic stimulator Vision Monitor MonPack 120 by Metrovision (Pérenchies, France): stimulus field size 23.6 arc deg centered on the fovea, contrast $100 \%$, mean luminance $50 \mathrm{~cd} /$ $\mathrm{m} 2$, and 2 reversals/s. The VEP were elicited by checkerboard stimuli with large (120') (106 min arc), medium $\left(60^{\prime}\right)$ (59 min arc), and small (15') (15.4 min arc) checks. The waveform of the transient examination was obtained at low temporal frequencies of the stimuli; then the values of the amplitude and latency (or time-to-peak) of P100 peaks were studied. A minimum of two recordings of each VEP condition were acquired, measured and displayed. After the recording, we manually analyzed 3 peaks N75, P100 and N135, each characterized by an amplitude and latency. The following results refer to the amplitude and latency of mean peak P100. The whole recording of pVEPs was performed adhering to ISCEV standards. ${ }^{21}$

Statistical analysis was performed with Excel for Windows. Descriptive statistical analysis was performed for all collected data. For each quantitative variable, mean, standard deviation and median were calculated. An ANOVA analysis was performed to test the hypothesis of equal means between the two groups, for each variable separately. The correlation matrix was calculated and, due to a high correlation between the measures, a p-value $<0.01$ was considered statistically significant. The assumption of equal variance in the two groups is not always evident, also from the inspection of the boxplots for the 6 variables. Hence, a $t$-test with not-equal variances (heteroscedastic) was applied in order to confirm ANOVA results (data not shown). The number of observations in each
Table I Ocular Abnormalities in the Gorlin-Goltz Syndrome (GGS) Group

\begin{tabular}{|l|c|}
\hline Ocular Abnormalities & Frequency in GGS Group \\
\hline Hypertelorism & $7 / 19$ \\
Strabismus & $8 / 19$ \\
Epiretinal membranes & $6 / 19$ \\
Myelinated fibers & $5 / 19$ \\
Congenital cataract & $3 / 19$ \\
Optic nerve coloboma & $1 / 19$ \\
Ptosis & $1 / 19$ \\
Palpebral cysts & $2 / 19$ \\
Anophthalmia & $1 / 19$ \\
\hline
\end{tabular}

group is large enough to ensure that the $t$-test is robust even if some deviations from the normality assumption is observed.

\section{Results}

The present study collected data from a total of 19 patients and 20 controls. The 2 groups were homogeneous for gender and age. Six eyes of 6 NBCCS patients had interfering conditions and were excluded from the statistical analysis. The ophthalmological examination highlighted several ocular abnormalities described in Table 1. The most common conditions were hypertelorism, strabismus, and epiretinal membranes. However, the ocular abnormalities (Figure 1) of the patients examined did not cause subjective disturbances in visual acuity. Visual acuity of all subjects included in this study was $\geq 10 / 10$ (logMAR $\leq 0.00$ ) with refractive error not exceeding 4 diopters. The results obtained after the recording of pVEPs are shown in Table 2. For each check size to $120^{\prime}, 60^{\prime}$ and $15^{\prime}$ were evaluated mean, median, and SD for amplitude and latency of the P100 component. The boxplots comparing the distribution for each studied parameter are available in Supplementary Figure S1. We reported a statistically significant reduction in amplitude and an increase in latency of the P100 component in the NBCCS group when compared to the CG with both ANOVA and $t$-test. (Table 2)

\section{Discussion}

The objective of the present study was to examine the macular pathway function in NBCCS patients without any significant ocular impairment confronting the results with a CG. To the best of our knowledge, this is the first VEP study to involve a relatively large number of patients (19 patients) in this rare condition. It is known that physiological factors such as age, gender, macular status, pupil 

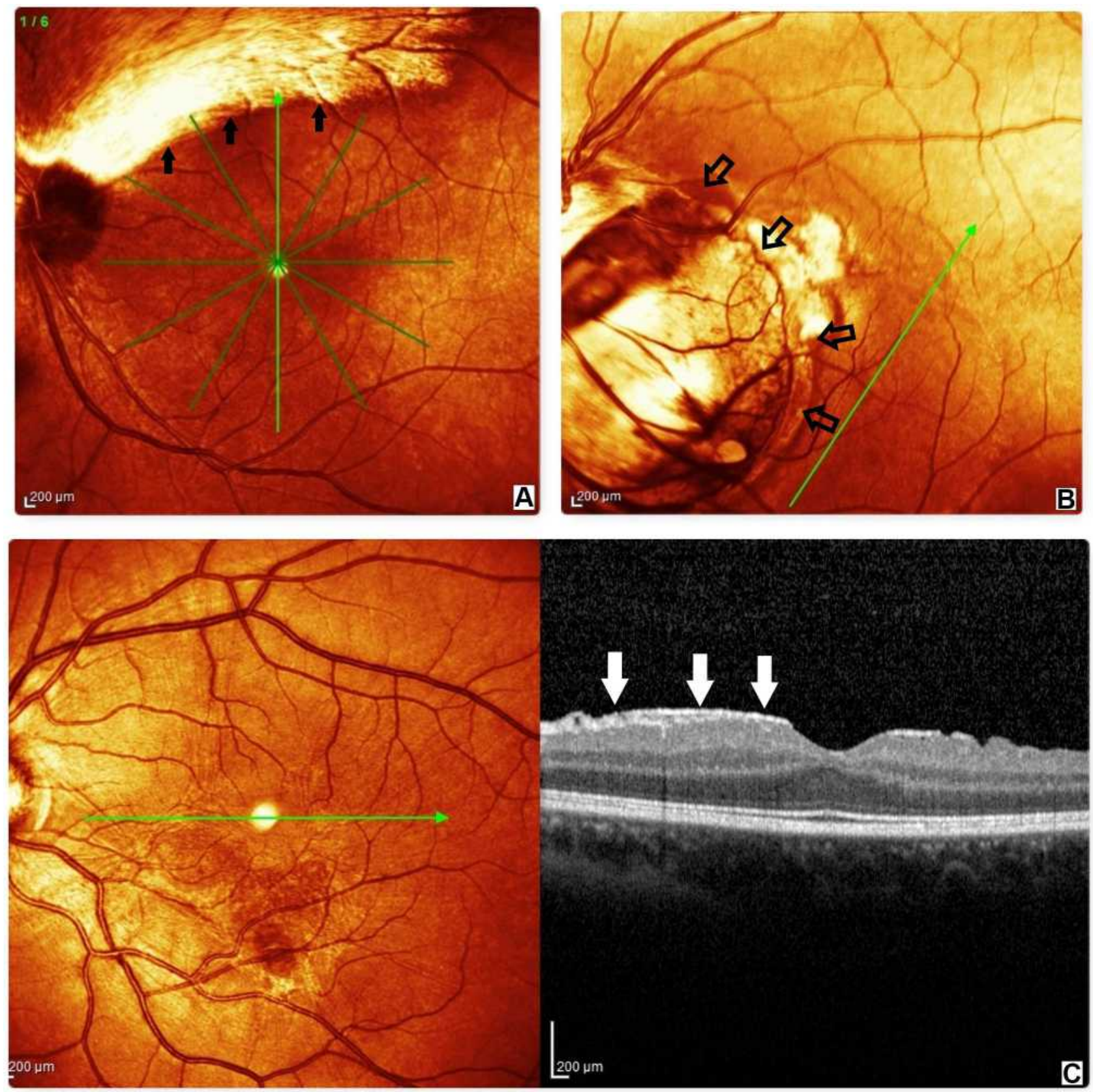

Figure I Spectral domain-optical coherence tomography (SD-OCT) findings in nevoid basal cell carcinoma syndrome (NBCCS). Patients with retinal alterations have been excluded from the pattern visual evoked potentials (pVEPs) measurement. (A) myelinated fibers (black full arrows); (B) optic nerve coloboma (empty black arrows); (C) on the right, sagittal scan of the epiretinal membrane (white arrows), on the left, near infrared image of epiretinal membrane.

diameter, refractive errors, type of stimulus, electrode position and anatomical factors may affect $p$ VEPs. To try to minimise the interference of these factors, the two groups were matched by age and sex, and strict exclusion criteria were applied. ${ }^{21-23}$

In this research, the analysis of VEP demonstrated an increase in latency as well as a reduction in amplitude in the observed patient, indicative of a qualitative and quantitative disorder of the optic pathway conduction (Figure 2). ${ }^{19,24}$ The significant results, throughout all frequencies of spatial stimulation, suggest a dysfunction of the macular pathway in NBCCS patients compared to the CG.

It is known that myelin involvement in numerous diseases determines an alteration in VEP P100 wave latency and amplitude. The ongoing VEP analysis of optic neuritis in patients suffering from multiple sclerosis (MS) showed an increase latency of P100 wave with normal amplitude. ${ }^{25,26}$ 
Table 2 Mean, Median and Standard Deviation (SD) for Each Check Size of Stimulation

\begin{tabular}{|l|c|c|c|c|c|c|c|}
\hline & \multicolumn{3}{|c|}{ Gorlin-Goltz (n = 3I Eyes) } & \multicolumn{3}{c|}{ Controls (n = 40 Eyes) } & ANOVA \\
\cline { 2 - 7 } & Mean & Median & SD & Mean & Median & SD \\
\hline PVEPI20 ampl & 11.6 & 11.3 & 4.4 & 15.8 & 15.2 & 5.0 & $<0.01$ \\
PVEPI20 lat & 114.1 & 112.5 & 13.3 & 102.1 & 102.5 & 3.7 & $<0.01$ \\
PVEP60 ampl & 11.1 & 10.6 & 4.2 & 16.6 & 16.8 & 6.0 \\
PVEP60 lat & 114.5 & 111.0 & 17.0 & 102.2 & 102.8 & 3.1 & $<0.01$ \\
PVEPI5 ampl & 11.5 & 10.4 & 4.6 & 19.0 & 19.0 & 5.6 & $<0.01$ \\
PVEPI5 lat & 127.2 & 121.3 & 20.8 & 111.2 & 110.8 & 6.7 & $<0.01$ \\
\hline
\end{tabular}

Notes: Amplitude is expressed in microvolts $=\mu \mathrm{V}$, and latency in milliseconds $=\mathrm{ms}$. Significant results in bold.

Abbreviations: Ampl, amplitude; Lat, latency; SD, standard deviation.

Interestingly, the amplitude reduction and the significant increase of the latency in NBCCS group in the transmission time of the optical impulse may correlate to a subclinical myelin alteration in NBCCS. ${ }^{14,19}$ Besides, we know that from a genetic point of view the activation of the SHH pathway is required for the development of the visual system. In fact, SHH expression is required for the induction of the optic nerve as well as photoreceptor differentiation. ${ }^{27}$
It has been demonstrated that the inactivation of the PATCH gene may be responsible for the development of fibroglial epiretinal membrane and abnormal ganglion-cell axon myelination. ${ }^{18}$

This is also shown by the common recurrence in our NBCCS group of epiretinal membranes and myelinated fibers. PATCH deficient murine models showed dysplastic foci in the retina and abnormal gliotic response further confirming the key role of SHH/PATCHED in glial cells
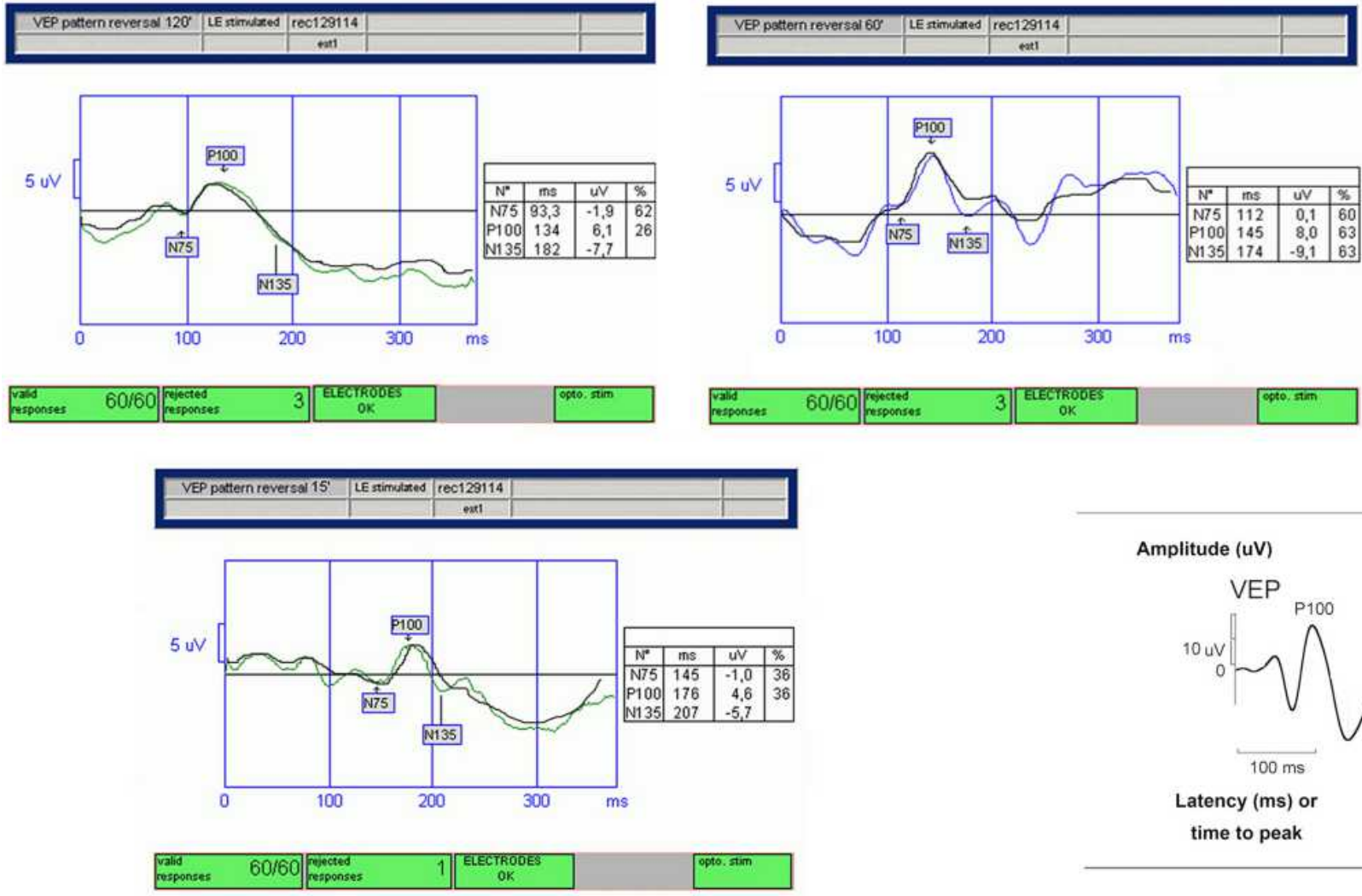

Amplitude (uV)

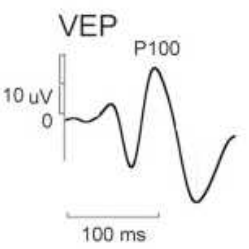

Latency (ms) or

time to peak

Figure 2 Transient pattern visual evoked potentials (p-VEPs) in Gorlin-Goltz syndrome (GGS) patient. Values with increased latency and reduced amplitude of the PI00 components, obtained for each spatial stimulation frequency $\left(120^{\prime}, 60^{\prime}\right.$, and $\left.15^{\prime}\right)$. Diagram of normal transient VEP illustrating how measurements of negative and positive waves were made (bottom right). 
homeostasis. ${ }^{14}$ Moreover, $\mathrm{SHH}$ is responsible for the development of oligodendrocytes progenitors and their differentiation into mature oligodendrocytes. ${ }^{28}$ These evidences suggest that the results obtained through the pVEPs may be caused by altered myelination at a subclinical level during the organogenesis.

The observed electrophysiological alterations could be determined also by other common manifestations of GGS. Dysgenesis/agenesis of the corpus callosum has been reported in around $10 \%$ off GGS patients. ${ }^{13}$ Corpus callosum is responsible for the interhemispheric connection in the brain including the occipital lobe where V1 is located. $^{29}$ Moreover, the integrity of corpus callosum is necessary for the development and plasticity of the visual system. ${ }^{30}$ Rugg et al studied the callosal function through VEPs elicited by lateralized stimuli in two patients affected by callosal agenesis. They found that VEPs from ipsilateral (to the visual field of stimulus) hemisphere were aberrant compared to contralateral, suggesting that VEPs for lateralized stimuli could be used to detect suspected callous disease. Therefore, subclinical alterations in the development of this anatomic structure may be partially responsible for the observed results. ${ }^{31}$

Other relatively common alterations in GGS such as falx cerebri calcification, bridging of sella turcica or cavum septum pellucidum could be also participate to the development of altered central conduction of the macular pathway function. Unfortunately, we were not able to find pre-existing literature on the role of these conditions. The association remains, therefore, purely speculative as magnetic resonance imaging (MRI) has not been yet performed on these patients; in the future this study will be a further object of evaluation for our research.

One consideration that we would still to discuss in patients with GGS is addressed to the dysmorphism linked to the congenital malformations of the skull which often presents with greater head circumference. This defect is associated with characteristic facies with frontal bossing, coarse facies, cleft lip, or palate, and moderate or severe hypertelorism.

Such anomalies could cause impaired functioning of the visual apparatus, as they have been described in the literature for other genetic conditions linked to cranial and cerebral malformations. ${ }^{32,33}$

The cause could also be traced to an altered path and/or an extension of the optical pathways beyond the norm. Or the craniofacial malformations could be the primary cause responsible for abnormal ganglion-cell axon myelination, or the lack of integrity of corpus callosum necessary for the development and plasticity of the visual system. Moreover, also the falx cerebri calcifications and the alterations of the bridging of sella turcica or cavum septum pellucidum could be caused by the craniofacial malformations. The discussion becomes complex as the cranial and brain morphological anomalies could both coincide and cause each other. Many studies will have to investigate in detail these morphogenetic and functional aspects related to the hereditary pathologies of the Gorlin-Goltz syndrome. In addition, another parameter to be considered is whether such craniofacial malformations are associated with an increase in Intracranial Pressure (ICP), a factor capable of determining abnormal VEPs. None of our patients showed evidence of papilledema, but that alone does not rule out the possibility of increased ICP. ${ }^{34}$ Some of these concepts will be evaluated in future studies on patients examined by us through careful evaluation together with colleagues neuroradiologists.

In conclusion, due to the lack of preexisting literature on the specific topic, only hypotheses can be made on the obtained results. Further morphological, functional, and laboratory studies will be needed to correlate the present results with the common brain affections found in GGS patients.

\section{Abbreviations}

GGS, Gorlin-Goltz syndrome; NBCCS, nevoid basal cell carcinoma syndrome; $p$ VEPs, pattern visual evoked potentials; SHH, Sonic Hedgehog; SUFU, Suppressor of the fused gene; BCC, basal cells carcinoma; ERM, epiretinal membrane.

\section{Ethical Approval}

All procedures performed in studies involving human participants were in accordance with the ethical standards of the institutional and/or national research committee and with the 1964 Helsinki Declaration and its later amendments or comparable ethical standards.

\section{Informed Consent}

Informed consent was obtained from all individual participants included in the study.

\section{Funding}

No funding was received for this research. 


\section{Disclosure}

All authors report no conflicts of interest for this work and certify that they have no affiliations with or involvement in any organization or entity with any financial interest (such as honoraria; educational grants; participation in speakers' bureaus, membership, employment, consultancies, stock ownership, or other equity interest; and expert testimony or patent licensing arrangements), or non-financial interest (such as personal or professional relationships, affiliations, knowledge or beliefs) in the subject matter or materials discussed in this manuscript.

\section{References}

1. Gorlin R, Goltz R. Multiple nevoid basal-cell epithelioma, jaw cysts and bifid rib. A syndrome. $N$ Engl J Med. 1960;262:908-912. doi:10.1056/NEJM196005052621803

2. Lo Muzio L. Nevoid basal cell carcinoma syndrome (Gorlin syndrome). Orphanet J Rare Dis. 2008;3:32. doi:10.1186/17501172-3-32

3. Endo M, Fujii K, Sugita K, Saito K, Kohno Y, Miyashita T. Nationwide survey of nevoid basal cell carcinoma syndrome in Japan revealing the low frequency of basal cell carcinoma. Am $J$ Med Genet A. 2012;158A(2):351-357. doi:10.1002/ajmg.a.34421

4. Fujii K, Miyashita T. Gorlin syndrome (nevoid basal cell carcinoma syndrome): update and literature review. Pediatr Int. 2014;56 (5):667-674. doi:10.1111/ped.12461

5. Bholah Z, Smith MJ, Byers HJ, Miles EK, Evans DG, Newman WG. Intronic splicing mutations in PTCH1 cause Gorlin syndrome. Fam Cancer. 2014;13(3):477-480. doi:10.1007/s10689-014-9712-9

6. Adolphe C, Hetherington R, Ellis T, Wainwright B. Patched1 functions as a gatekeeper by promoting cell cycle progression. Cancer Res. 2006;66(4):2081-2088. doi:10.1158/0008-5472.CAN-05-2146

7. Sasai N, Toriyama M, Kondo T. Hedgehog Signal and Genetic Disorders. Front Genet. 2019;10:1103. doi:10.3389/fgene.2019.01103

8. Cherry AL, Finta C, Karlström M, et al. Structural basis of SUFU-GLI interaction in human Hedgehog signalling regulation. Acta Crystallogr D Biol Crystallogr. 2013;69(Pt 12):2563-2579. doi:10.1107/S0907444913028473

9. Smith MJ, Beetz C, Williams SG, et al. Germline mutations in SUFU cause Gorlin syndrome-associated childhood medulloblastoma and redefine the risk associated with $\mathrm{PTCH} 1$ mutations. J Clin Oncol. 2014;32(36):4155-4161. doi:10.1200/JCO.2014.58.2569

10. Evans DG, Ladusans EJ, Rimmer S, Burnell LD, Thakker N, Farndon PA. Complications of the naevoid basal cell carcinoma syndrome: results of a population based study. J Med Genet. 1993;30(6):460-464. doi:10.1136/jmg.30.6.460

11. Kiwilsza M, Sporniak-Tutak K. Gorlin-Goltz syndrome-a medical condition requiring a multidisciplinary approach. Med Sci Monit. 2012;18(9):RA145-RA153. doi:10.12659/msm.883341

12. Bresler SC, Padwa BL, Granter SR. Nevoid Basal Cell Carcinoma Syndrome (Gorlin Syndrome). Head Neck Pathol. 2016;10(2):119124. doi:10.1007/s12105-016-0706-9

13. Kimonis VE, Mehta SG, Digiovanna JJ, Bale SJ, Pastakia B. Radiological features in 82 patients with nevoid basal cell carcinoma (NBCC or Gorlin) syndrome. Genet Med. 2004;6(6):495-502. doi:10.1097/01.gim.0000145045.17711.1c

14. Black GC, Mazerolle CJ, Wang Y, et al. Abnormalities of the vitreoretinal interface caused by dysregulated Hedgehog signaling during retinal development. Hum Mol Genet. 2003;12(24):3269-3276. doi: $10.1093 / \mathrm{hmg} / \mathrm{ddg} 356$
15. Shanley S, Ratcliffe J, Hockey A, et al. Nevoid basal cell carcinoma syndrome: review of 118 affected individuals. Am J Med Genet. 1994;50(3):282-290. doi:10.1002/ajmg.1320500312

16. Moramarco A, Himmelblau E, Miraglia E, et al. Ocular manifestations in Gorlin-Goltz syndrome. Orphanet $J$ Rare Dis. 2019;14 (1):218. doi:10.1186/s13023-019-1190-6

17. Lo Muzio L, Nocini PF, Savoia A, et al. Nevoid basal cell carcinoma syndrome. Clinical findings in 37 Italian affected individuals. Clin Genet. 1999;55(1):34-40. doi:10.1034/j.1399-0004.1999.5501 06. $\mathrm{x}$

18. Chen JJ, Sartori J, Aakalu VK, Setabutr P. Review of ocular manifestations of nevoid basal cell carcinoma syndrome: what an ophthalmologist needs to know. Middle East Afr J Ophthalmol. 2015;22 (4):421-427. doi:10.4103/0974-9233.167815

19. Romano M, Iacovello D, Cascone NC, Contestabile MT. Identification of a novel mutation in the $\mathrm{PTCH}$ gene in a patient with Gorlin-Goltz syndrome with unusual ocular disorders. Eur J Ophthalmol. 2011;21(4):516-519. doi:10.5301/ EJO.2010.6186

20. Kimonis VE, Goldstein AM, Pastakia B, et al. Clinical manifestations in 105 persons with nevoid basal cell carcinoma syndrome. Am J Med Genet. 1997;69(3):299-308.

21. Odom JV, Bach M, Brigell M, et al. ISCEV standard for clinical visual evoked potentials: (2016 update). Doc Ophthalmol. 2016;133 (1):1-9. doi:10.1007/s10633-016-9553-y

22. Emmerson-Hanover R, Shearer DE, Creel DJ, Dustman RE. Pattern reversal evoked potentials: gender differences and age-related changes in amplitude and latency. Electroencephalogr Clin Neurophysiol. 1994;92(2):93-101. doi:10.1016/0168-5597(94)900 49-3

23. Bemelmans NA, Tilanus MA, Cuypers MH, Pinckers AJ. Patternreversal visual evoked potentials in patients with epiretinal membrane. Am J Ophthalmol. 1997;123(1):97-102. doi:10.1016/ s0002-9394(14)70998-5

24. Fishman GA, Birch DG, Holder GE, Brigell MG. Electrophysiologic Testing in Disorders of the Retina, Optic Nerve, and Visual Pathway. 2nd ed. San Francisco, United States: American Academy of Ophthalmology Monograph Series; 2002.

25. Gareau PJ, Gati JS, Menon RS, et al. Reduced visual evoked responses in multiple sclerosis patients with optic neuritis: comparison of functional magnetic resonance imaging and visual evoked potentials. Mult Scler. 1999;5(3):161-164. doi:10.1177/13524585 9900500304 PMID: 10408715.

26. Neto SP, Alvarenga RM, Vasconcelos CC, Alvarenga MP, Pinto LC, Pinto VL. Evaluation of pattern-reversal visual evoked potential in patients with neuromyelitis optica. Mult Scler. 2013;19(2):173-178. doi:10.1177/1352458512447597

27. Dakubo GD, Wang YP, Mazerolle C, Campsall K, McMahon AP, Wallace VA. Retinal ganglion cell-derived sonic hedgehog signaling is required for optic disc and stalk neuroepithelial cell development. Development. 2003;130(13):2967-2980. doi:10.124 2/dev.00515

28. Tekki-Kessaris N, Woodruff R, Hall AC, et al. Hedgehog-dependent oligodendrocyte lineage specification in the telencephalon. Development. 2001;128(13):2545-2554.

29. Dougherty RF, Ben-Shachar M, Bammer R, Brewer AA, Wandell BA. Functional organization of human occipital-callosal fiber tracts. Proc Natl Acad Sci US A. 2005;102(20):7350-7355. doi:10.1073/pnas.0500003102

30. Pietrasanta M, Restani L, Caleo M. The corpus callosum and the visual cortex: plasticity is a game for two. Neural Plast. 2012; 2012:838672. doi:10.1155/2012/838672

31. Rugg MD, Milner AD, Lines CR. Visual evoked potentials to lateralised stimuli in two cases of callosal agenesis. J Neurol Neurosurg Psychiatry. 1985;48(4):367-373. doi:10.1136/jnnp.48.4. 367 
32. Haredy MM, Liasis A, Davis A, et al. Serial, visually-evoked potentials for the assessment of visual function in patients with craniosynostosis. J Clin Med. 2019;8(10):1555. doi:10.3390/jcm81 01555

33. Coupland SG, Sarnat HB. Visual and auditory evoked potential correlates of cerebral malformations. Brain Dev. 1990;12(5):46 6-472. doi:10.1016/s0387-7604(12)80209-9
34. Haredy MM, Liasis A, Fu V, et al. Serial Visual Evoked Potentials in Patients with Craniosynostosis and Invasive Intracranial Pressure Monitoring. Plast Reconstr Surg. 2019;144(3):446e-452e. doi:10. 1097/PRS.0000000000005935

\section{Publish your work in this journal}

Eye and Brain is an international, peer-reviewed, open access journal focusing on clinical and experimental research in the field of neuroophthalmology. All aspects of patient care are addressed within the journal as well as basic research. Papers covering original research, basic science, clinical and epidemiological studies, reviews and

Submit your manuscript here: https://www.dovepress.com/eye-and-brain-journal evaluations, guidelines, expert opinion and commentary, case reports and extended reports are welcome. The manuscript management system is completely online and includes a very quick and fair peerreview system, which is all easy to use. Visit http://www.dovepress. com/testimonials.php to read real quotes from published authors. 